“드 2013 IEEE. Personal use of this material is permitted. Permission from IEEE must be obtained for all other uses, in any current or future media, including reprinting/republishing this material for advertising or promotional purposes, creating new collective works, for resale or redistribution to servers or lists, or reuse of any copyrighted component of this work in other works." 


\section{B-H Relations of Magnetorheological Fluid under 2- D Rotating Magnetic Field Excitation}

\author{
Youguang Guo, Jianbin Zeng, Jianguo Zhu, Haiyan Lu \\ Faculty of Engineering and Information Technology \\ University of Technology, Sydney \\ Sydney, Australia \\ Youguang.Guo-1@uts.edu.au, Jianbin.Zeng@uts.edu.au, \\ Jianguo.Zhu@uts.edu.au, Haiyan.Lu@uts.edu.au
}

\begin{abstract}
This paper presents the investigation of the B-H relations of a magnetorheological (MR) fluid under onedimensional (1-D) alternating and two-dimensional (2-D) rotating magnetic field excitations, where $B$ is magnetic flux density and $H$ is magnetic field strength. The measurement is carried out by using a single sheet tester with an MR fluid sample. The measurement principle and structure of the testing system are described. The calibration of the $B$ and $H$ sensing coils are also reported. The relations between $B$ and $H$ on the MR fluid sample under 2-D rotating magnetic field excitations have been measured and compared with the results under 1-D excitations, showing that the B-H relations under 2-D excitations are significantly different from the 1-D case. These data would be useful for design and analysis of MR smart structures like MR dampers.
\end{abstract}

Keywords-magnetic field strength (H); flux density (B); B-H relation; magnetorheological fluid; rotating magnetic field

\section{INTRODUCTION}

The magneto-rheological (MR) material is a kind of controllable or magnetic field responsive smart material. In general, it is in the form of fluid of dispersions of non-colloidal and polarizable particles in a viscous non-magnetic carrier. Its rheology can be controlled by an external magnetic field. Under the influence of an applied magnetic field, this remarkable material can reversibly change its state within milliseconds between linear viscous liquids that are freeflowing and semisolids that have controllable yield strength. Particularly, the yield stress of the fluid can be controlled accurately by varying the magnetic field intensity.

This promising feature makes the MR material smart, simple, quiet, and capable of a rapid interface between an electronic control system and a mechanical system. The MR material has been widely used as a smart material in various devices. Shock absorbers, vibration dampers and prosthetic devices are the examples of exciting applications of the MR material concerning semi-active control of vibrations or torque transfer [1-6].

Because of its unique properties, the MR material has attracted great research interest in new material development, rheological mechanism, property measurement and modeling, as well as new applications [7-11]. However, the properties and

\author{
Jianxun Jin \\ Center of Applied Superconductivity and Electrical \\ Engineering \\ University of Electronic Science and Technology of China \\ Chengdu, China \\ jxjin@uestc.edu.cn
}

mechanism of MR fluid material have not been deeply understood, so the applications of the material in industry have been limited in only several areas like dampers and vibration absorbers. For innovative design and application of the material, proper understanding of mechanism and property modeling are fundamental and crucial.

This paper presents some of our investigation on the magnetic hysteresis property, i.e. the relation between B (magnetic flux density) and $\mathrm{H}$ (magnetic field strength), of MR fluid material under one-dimensional (1-D) alternating and two-dimensional (2-D) rotating magnetic field excitations by using a single sheet tester [12-15]. The structure and measuring principle of the tester and calibration of the $\mathrm{B}$ and $\mathrm{H}$ sensing coils are described. Some measurements, such as the B-H hysteresis loop under 1-D alternating magnetic excitation and B-H loci under 2-D rotational excitation, are reported. These results would be helpful for deeper understanding of the MR properties and the design of smart devices using MR materials.

\section{2-D MAGANETIC PROPERTY Testing SYSTEM}

\section{A. Single Sheet 2-D Magnetic Tester}

A single sheet 2-D magnetic tester [12-15], developed at the University of Technology, Sydney, as shown in Fig. 1, has been modified to measure the magnetic hysteresis properties of the MR fluid under 1-D alternating and 2-D rotational magnetic fields. Supported by a computerized digital signal processing system, the tester can measure B-H relationships and core losses with either alternating magnetic fluxes in any specified orientation in 1-D cases or circular or elliptical rotating fluxes with any specified axis ratio in 2-D cases.

The magnetic field in the sample, which is at the center of the tester, is produced by two groups of excitation coils placed on the $\mathrm{X}$-axis and $\mathrm{Y}$-axis, respectively. The excitation voltages and currents for the excitation coils are provided by two identical power amplifiers. By controlling the waveforms, magnitudes and phase angles of the excitation voltages on the $\mathrm{X}$ and $\mathrm{Y}$ coils, any complex 1-D or 2-D magnetic flux density vector, such as an alternating magnetic flux density inclined at a specified angle from the $\mathrm{X}$ or $\mathrm{Y}$ axis, a circular or elliptical rotating magnetic flux density in a 2-D plane, or a rotating magnetic flux density of any specified locus, can be obtained. 


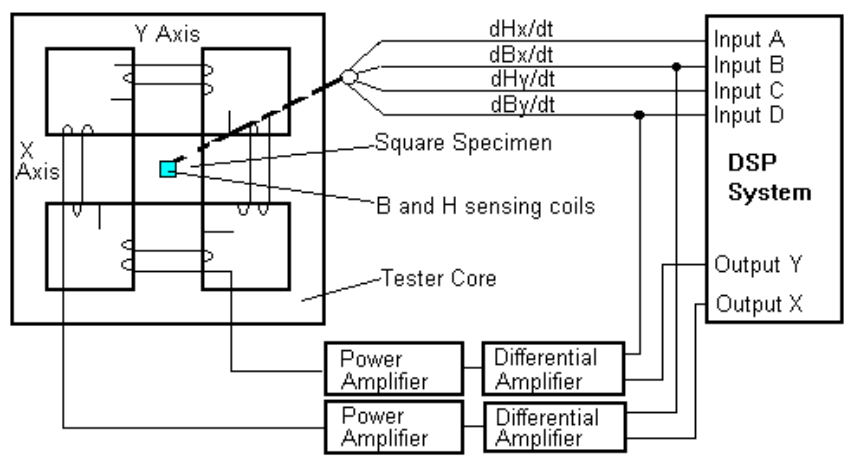

Figure 1. Schematic diagram of a 2-D magnetic property testing system

\section{B. MR Fluid Sample}

Fig. 2 shows the photo of the 2-D magnetic tester with the MR sample in the center. A rectangle MR fluid sample container, which is made of nonmagnetic Plexiglas material with four mild steel magnetic poles embedded along the four sides by the authors, is shown in Fig. 3, where the MR fluid was mainly contained as a circular disk within the square container. The magnetic poles of the tester have been modified so that a uniform magnetic field distribution in the MR fluid could be obtained.

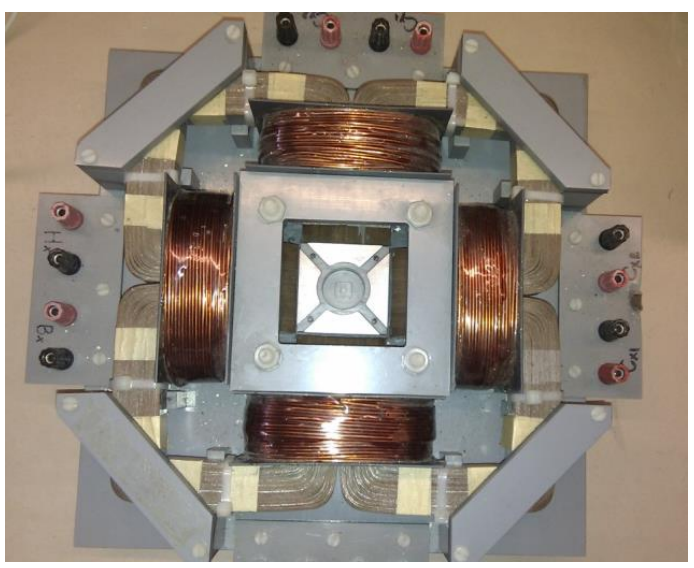

Figure 2. Photo of the single sheet tester

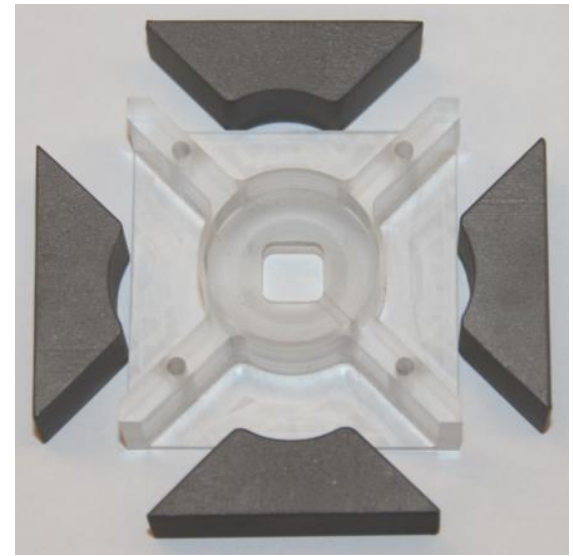

Figure 3. MR fluid sample container
Fig. 4(a) shows the photo of the MR fluid (MRF) sample and Fig. 4(b) indicates the positions of the $\mathrm{B}$ and $\mathrm{H}$ sensing coils. H sensing coils are placed between the two halves of the MRF sample to measure the magnetic field strength $(\mathrm{H})$, and $\mathrm{B}$ sensing coils are placed inside the sample for measuring the flux density (B). The $\mathrm{H}$ and $\mathrm{B}$ sensing coils can be calibrated by using a long solenoid and a high-precision Gaussian meter. Fig. 5 illustrates the diagram of the solenoid, where $\mathrm{A}$ is the solenoid frame, B the coils to be calibrated, $\mathrm{C}$ the Gauss meter probe, $\mathrm{D}$ the connector for the sensing coil output terminals, $\mathrm{E}$ the turn-table for mounting the coils, and $F$ and $G$ are the wheel rubber band for adjusting the coil orientation. Fig. 6 shows the photo of the solenoid in which the $\mathrm{H}$ and $\mathrm{B}$ coils are put for calibration, while Fig. 7 shows the turn-table for holding the MRF sample.

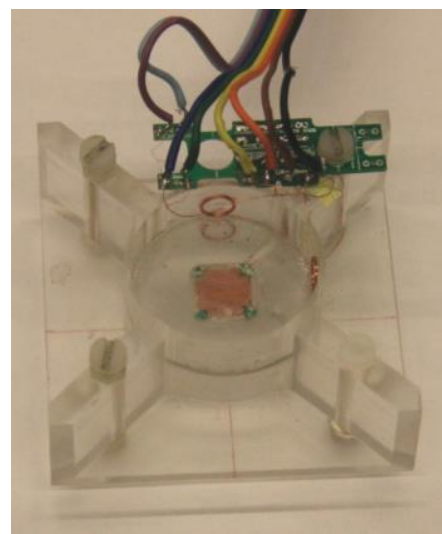

(a)

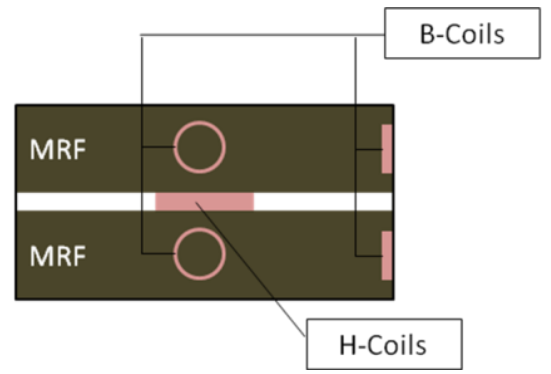

(b)

Figure 4. MRF sample with sensing coils: (a) Photo; (b) Illustration showing the positions of the $\mathrm{B}$ and $\mathrm{H}$ sensing coils

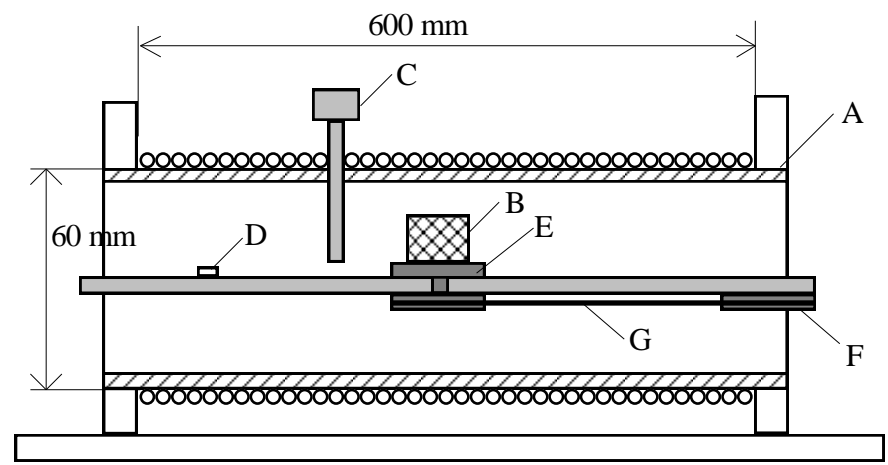

Figure 5. Solenoid for calibration of $\mathrm{H}$ and $\mathrm{B}$ sensing coils 


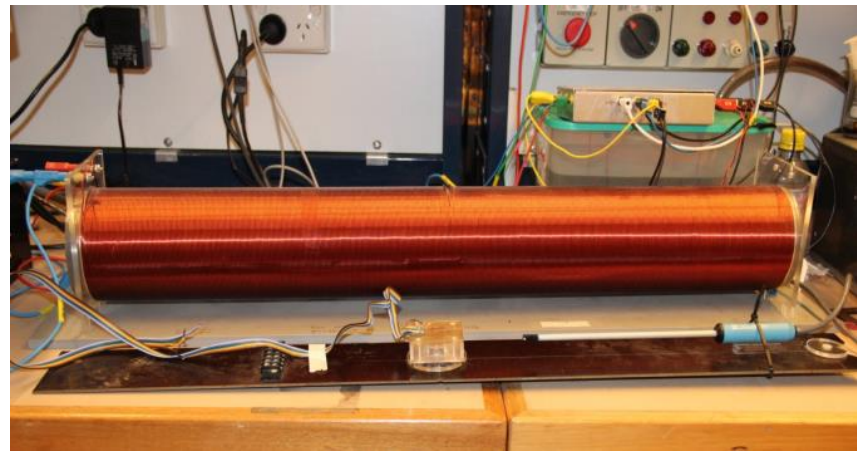

Figure 6. Photo of the solenoid for sensing coil calibration

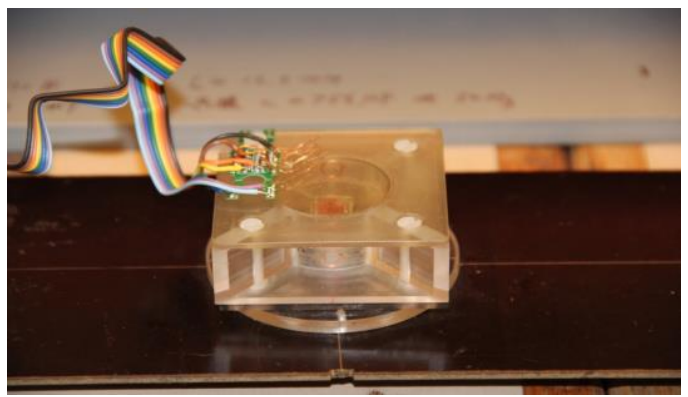

Figure 7. Photo of the turn-table for holding the MRF sample

\section{MAGNETIC MEASUREMENT OF MR FLUID}

The surface magnetic field strength along one axis, e.g. the $\mathrm{x}$-axis or the $\mathrm{y}$-axis, can be computed from the induced output voltage of the coil along that axis:

$$
H_{i}=\frac{1}{\mu_{o} K_{H i}} \int V_{H i} d t \quad(i=x, y)
$$

where $V_{H i}$ is the output voltage of the coil, $\mu_{o}$ the permeability of air, and $K_{H i}$ the coil coefficients, which are determined by the calibration solenoid as $K_{H x}=0.00109, K_{H y}=0.00147$.

The flux density on one axis can be calculated by

$$
B_{i}=\frac{1}{N_{B i} A_{B i}} \int V_{B i} d t \quad(i=x, y)
$$

where $N_{B i}$ is the number of turns, $A_{B i}$ the cross-sectional area, and $V_{B i}$ the induced terminal voltage of the B-coil on the axis. The coefficients $K_{B i}=N_{B i} A_{B i}$ can also be determined by the calibration solenoid as $K_{B x}=0.00143, K_{B y}=0.00138$.

The B-H relationships of the MRF sample (MRF-132DG by Lord Corporation, USA) have been measured under various magnetic field excitations. For example, Fig. 8 plots the B-H loop under 1-D alternating excitation along the $\mathrm{x}$-axis at $10 \mathrm{~Hz}$, and Fig. 9 plots the B-H relation under 2-D rotational excitation while B loci are controlled to be circular. Fig. 10 shows the B-H relation under 2-D elliptically rotating magnetic field B, e.g. the phase difference between the currents in the $\mathrm{x}$ and $y$-axis excitation coils is controlled as $45^{\circ}$. It can be seen from Fig. 9 that when the B locus is circular, the corresponding $\mathrm{H}$ locus becomes non-circular due to magnetic saturation. For the same reason, it is also observed from Fig. 10 that the loci of $\mathrm{B}$ and $\mathrm{H}$ are not the same.

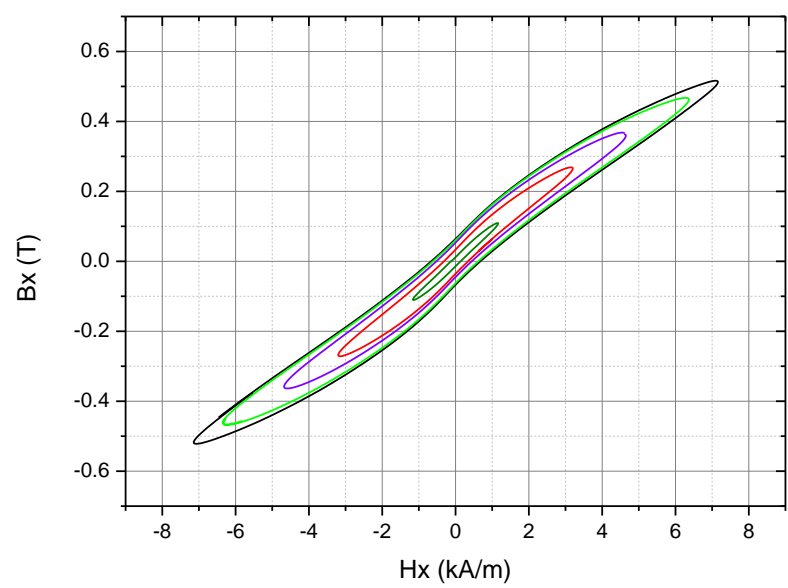

Figure 8. H-B loop of the MR sample under $10 \mathrm{~Hz}$ alternating sinusoidal magnetic flux density
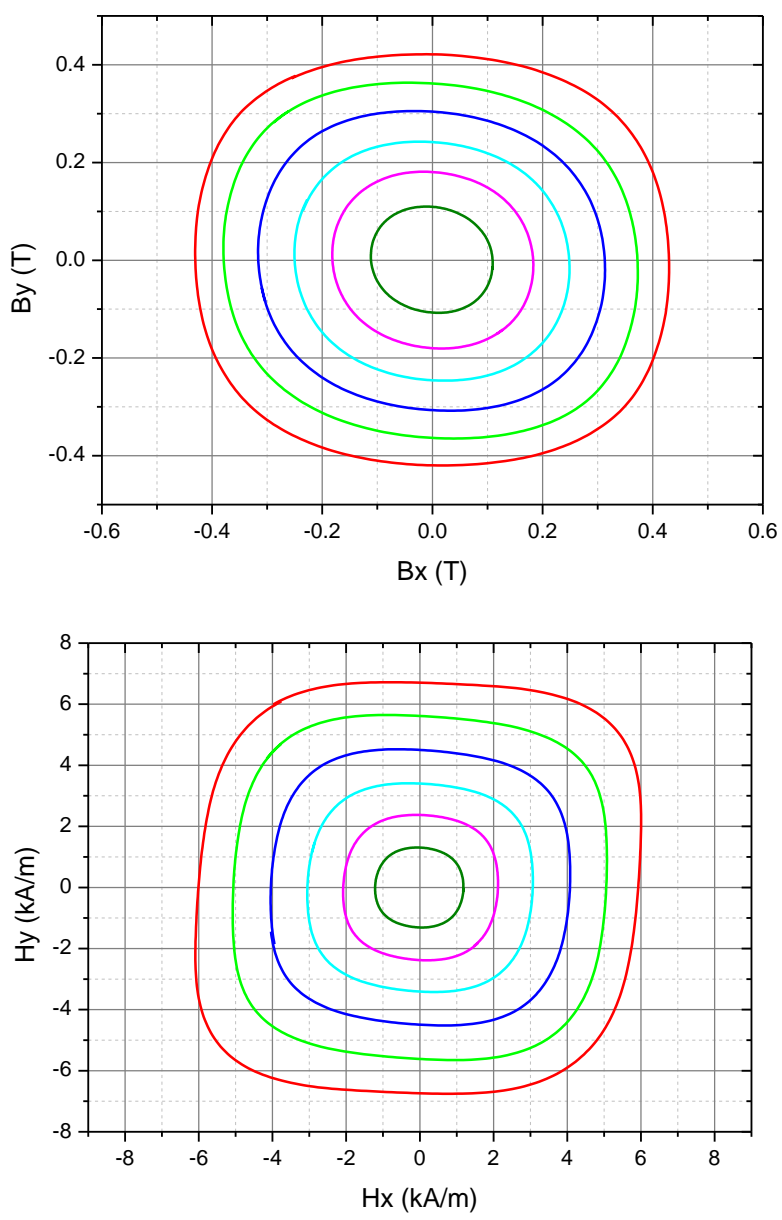

Figure 9. Loci of $\mathrm{B}$ and $\mathrm{H}$ when $\mathrm{B}$ is controlled circularly rotating at $10 \mathrm{~Hz}$ 

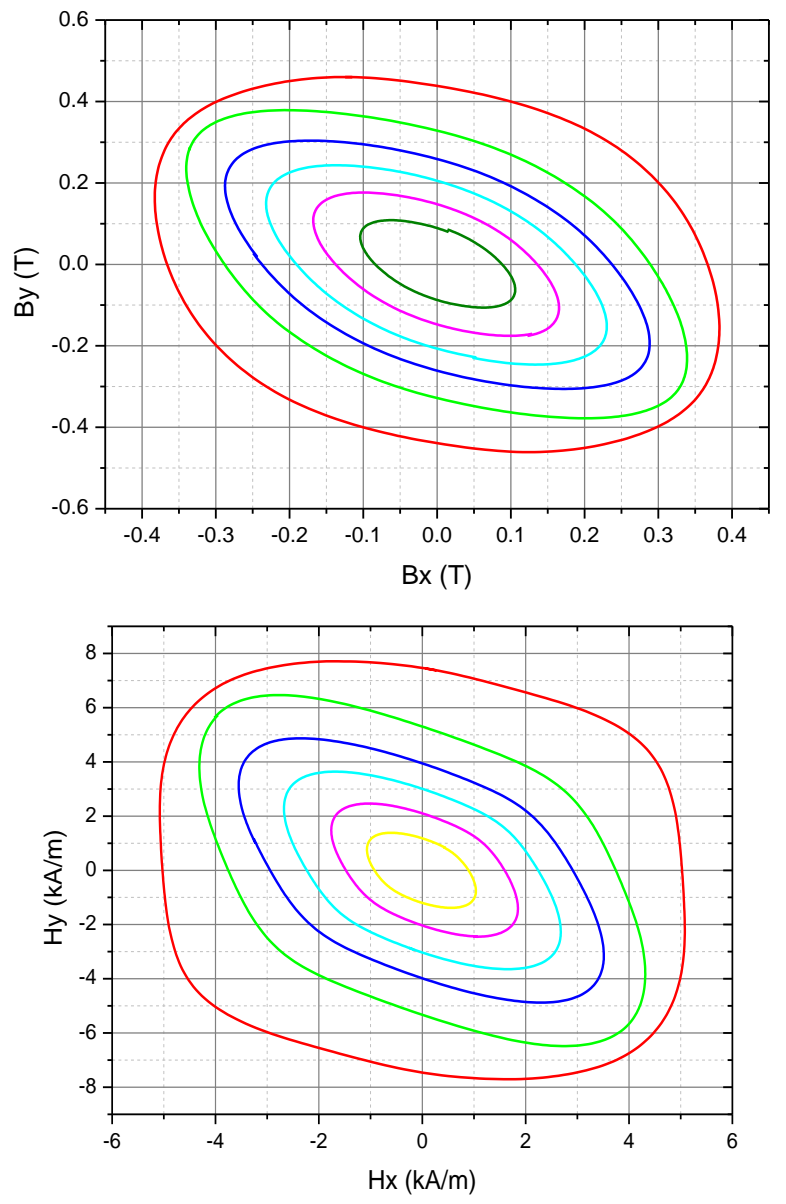

Figure 10. Loci of B and $\mathrm{H}$ when $\mathrm{B}$ is controlled elliptically rotating at $10 \mathrm{~Hz}$

\section{CONCUlUSION}

In this paper, the magnetic properties of an MR fluid sample are investigated with 1-D alternating and 2-D rotating magnetic field excitations by using a single sheet testing system. The measured B-H loops and B-H loci would be helpful for understanding of the properties and mechanism of the MR material and for designing MR devices.

\section{ACKNOWLEDGMENT}

The authors of the paper thank the Australian Research Council for the financial support under Discovery Project Grant Scheme (DP1093788).

\section{REFERENCES}

[1] J. Rabinow, Magnetic Fluid Torque and Force Transmitting Device, US Patent 2575360, 1951

[2] B. F. Spencer, S. J. Dyke, and M. K. Sain, "Magnetorheological dampers: a new approach to seismic protection of structures," Proc. $35^{\text {th }}$ Conf. on Decision and Control, Kobe, Japan, Dec. 1996, pp. 676-681.

[3] M. R. Jolly and J. W. Bender, "Properties and applications of commercial magnetorheological fluids," Proc. $5^{\text {th }}$ Annual Int. Symposium on Smart Structures and Materials, San Diego, USA, 1998.

[4] B. J. Bass and R. E. Christenson, "System identification of a $200 \mathrm{kN}$ magneto-rheological fluid damper for structural control in large-scale smart structures," Proc. American Control Conf., New York, USA, July 2007.

[5] S. Kikuchi and K. Hamamoto, "HAMA device - haptic display for immersive virtual environments," Proc. Int. Symp. on Communications and Information Technologies, Vientiane, Laos, Oct. 2008, pp 453-458.

[6] M. M. Rashid, N. A. Rahim, M. A. Hussain, and M. A. "Analysis and experimental study of magnetorheological-based damper for semiactive suspension system using fuzzy hybrids," IEEE Trans. Magn., vol. 47, pp 1051-1059, 2011.

[7] V. G. Bashtovoi, D. N. Kabachnikov, and G. Bossis, "Using of ferromagnetic particles suspension as controllable dissipative environment," Proc. $8^{\text {th }}$ Int. Conf. on Electro-Rheological Fluids and Magneto-Rheological Suspensions, Nice, France, 9-13 July 2001, pp 236-242.

[8] G. Bossis, S. Lacis, A. Meunier, and O. Volkova, "Magnetorheological fluids," Journal of Magnetism and Magnetic Materials, vol. 252, pp. 224-228, 2002.

[9] N. M. Wereley, "Adaptive magnetorheological crew seat suspensions for vibration and shock mitigation," Proc. $11^{\text {th }}$ Int. Conf. on ER Fluids and MR Suspensions, Dresden, Germany, Aug. 2008.

[10] W. H. Li, H. Du, and N. Q. Guo, "Dynamic behavior of MR suspensions at moderate flux densities," Materials Science and Engineering: A, vol. 371, pp 9-15, 2004.

[11] H. Chiriac and G. Stoian, "Influence of the particle size and size distribution on the magnetorheological fluids properties," IEEE Trans. Magn., vol. 45 pp. 4049-51, 2009.

[12] J. J. Zhong, J. G. Zhu, Y. G. Guo, and Z. W. Lin, "Improved measurement with $2 \mathrm{D}$ rotating fluxes considering the effect of internal field," IEEE Trans. Magn., vol. 41, pp. 3709-11, 2005.

[13] Y. G. Guo, J. G. Zhu, and J. J. Zhong, "Measurement and modeling of magnetic properties of soft magnetic composite materials under 2D vector magnetizations," Journal of Magnetism and Magnetic Materials, vol. 302, pp. 14-19, 2006.

[14] J. B. Zeng, Y. G. Guo, J. G. Zhu, and Z. W. Lin, "Magnetic hysteresis property of magneto-rheological fluid material under 2D fluxes," Proc. 2011 IEEE Int. Conf. on Applied Superconductivity and Electromagnetic Devices, Dec. 2011, Sydney, Australia, pp 256-259.

[15] Y. G. Guo, J. B. Zeng, J. G. Zhu, Z. W. Lin, and Y. C. Li, "Magnetic hysteresis of magnetorheological fluid under $2 \mathrm{D}$ rotating magnetic field excitation," Proc. 13 ${ }^{\text {th }}$ Int. Conf. on Electrorheological Fluids and Magnetorheological Suspensions, July 2012, Ankara, Turkey. 